\title{
3-S29-2 Symposium2
}

\section{Analysis of contractile functions of human iPS-derived cardiomyocytes using motion field imaging}

\author{
Junko Kurokawa, Kazuho Sakamoto, Masahiko Yamaguchi
}

Dept. Bio-Info. Pharmacol., Univ. Shizuoka

Human iPS cell-derived cardiomyocyte (hiPSC-CM) is conceptually promising as an unlimited source of human cardiomyocytes for cardiac pharmacological assessment including pre-clinical safety testing. However, intra- and interline variation in functional properties of hiPSC-CM remain to be solved completely. In order to improve the accuracy of pharmacological assessment, we conducted a multidisciplinary approach for developing new methods to evaluate effects of drugs on contractile functions. We aimed to increase throughput of pharmacological assessment for contractile functions of hiPSC-CMs using a motion field imaging which is a noninvasive assay system using high speed video image of hiPSC-CMs. The technique enabled us to obtain precise and stable quantitative values for contractile functions of hiPSC-CMs from single cells, and revealed a relationship between contractile function and molecular expression in hiPSC-CMs. The relationship was consistent with what we investigated in murine cardiac cells. We would like to discuss how the multidisciplinary approach can improve predictability of pharmacological/toxicological assessment for physiological functions of hiPSC-CMs. (JSPS KAKENHI JP17K19499, JP19H03380, AMED 19mk0104117) 